\title{
Glaucoma after penetrating keratoplasty: Risk factors and its management
}

\author{
Anuradha Raj ${ }^{1 *}$, Harsh Bahadur ${ }^{2}$ \\ ${ }^{\mathbf{1}}$ Associate Professor, ${ }^{2}$ Professor, Dept. of Ophthalmology, Swami Rama Himalayan University, HIMS, Dehradun, Uttarakhand, India \\ *Corresponding Author: Anuradha Raj \\ Email: dranuradha_sagar@yahoo.com
}

\begin{abstract}
Glaucoma is the second leading cause of graft failure after penetrating keratoplasty. Prompt diagnosis and aggressive management is mandatory to salvage the graft. Indications of penetrating keratoplasty, lens status, additional procedures with penetrating keratoplasty, preexisting glaucoma and re-graft are the main risk factors for glaucoma after penetrating keratoplasty. Various newer modalities like recent tonopens, ultrasound biomicroscopy, and anterior segment optic coherence tomography are helpful tools for its diagnosis. Various newer drugs, surgical modifications such as trabeculectomy with antimetabolites like mitomycin-C and 5 fluorouracil, glaucoma drainage devices, cyclodestructive procedures have added the chances of success of its management, but still graft failure secondary to glaucoma is very common.
\end{abstract}

Keywords: Trabeculectomy, Glaucoma drainage device, Graft failure.

\section{Introduction}

Glaucoma following penetrating keratoplasty (PK) has a relatively high frequency, it can appear both in early as well as late post-operative period of PK. It is very hard to diagnose glaucoma after PK. Even if intraocular pressure (IOP) remains high for longer duration, can lead to damage of endothelial cells. Medical or surgical treatment can also affect the fate of graft negatively. ${ }^{1}$ Graft rejection and high astigmatism are well known complications of PK. Glaucoma after PK leads to irreversible visual loss and is second leading cause for graft failure due to significant endothelial cell loss especially in patients who have already low endothelial reserve. ${ }^{2}$ Post-PK glaucoma (PPKG) is an elevation of IOP greater than $21 \mathrm{mmHg}$, independent of the optic nerve or visual field changes as it is difficult to perform in these cases because of high astigmatism and hazy graft. Tonometry is also difficult in these cases because of thick or astigmatic corneal graft. The definition of PPKG does not justify the glaucoma because of practical deficiencies and in cases of preexisting glaucoma it is difficult to define this.

Some studies enclose all these cases in PPKG, meanwhile others include only the cases that require escalation of antiglaucoma therapy to maintain IOP as medications, laser or surgical treatment. ${ }^{3}$

\section{Incidence of PPKG}

Different studies showed a variable incidence of PPKG, ranging from 9 to $31 \%$ early postoperatively and from 18 to $35 \%$ in the late postoperative period. Raj et al reported the overall incidence of raised IOP after PK was 32.25\%.,

\section{Various Risk Factors}

Recognition of risk factors is important for the prevention, diagnosis and early treatment of PPKG.

\section{Pre-operative and Intraoperative Risk Factors}

The most significant risk factors are preexisting glaucoma, lens status (i.e. aphakia, pseudophakia) and the disease for which PK is performed. Hemanth et al. compared the incidence of glaucoma after PK in phakic, pseudophakic, and aphakic eyes. The aphakic group had the highest risk, followed by the pseudophakic and phakic group; but no statistically significant difference was found between pseudophakic and phakic patients. ${ }^{3}$ Raj et al reported that subjects with age group $>40$ years, corneal opacity, increased recipient size and thicker grafts were risk factors for PPKG. ${ }^{5}$ Tight and superficial suturing with long bites, larger recipient bed with same size donor button, retained viscoelastic can cause PPKG. Karadog et al reported that traumatic corneal scar, graft thinning, graft infection, deep infective keratitis, bullous keratopathy and graft rejection were all high-risk factors. Those eyes with previous anterior segment surgery or had a pre-existing inflammatory condition had a higher chance of developing glaucoma postoperatively. ${ }^{6}$ Tight suturing, larger trephine sizes, long bites (more compressed tissue), excessive manipulation of iris and angles, iridoplasty, goniosynechiolysis, increased peripheral corneal thickness can also aggravate this condition. $^{7}$ (Fig. 1 a,b)

\section{Post-operative Risk Factors}

In early post-operative period various causes of raised IOP are inflammation, retained viscoelastic substances, hyphema, pupillary-block glaucoma, preexisting glaucoma in aphakic eyes and after additional procedures with PK like cataract surgery, Iris repair or pupillary reconstruction or vitrectomy. In addition to these factors long term topical steroid, graft rejection, ghost cell glaucoma, malignant glaucoma and chronic angle-closure glaucoma can be triggering factors for PPKG. In aphakic patients of PK, absence of lens makes relaxed posterior support and anterior support is lost due to incised descemet's membrane which leads to aqueous outflow obstruction due to trabecular complex collapse which eventually leads to $\mathrm{PPKG} .{ }^{8}$ Due to this fact in aphakic patients $1 \mathrm{~mm}$ oversized donor button graft reduce the incidence of PPKG. ${ }^{9}$ 


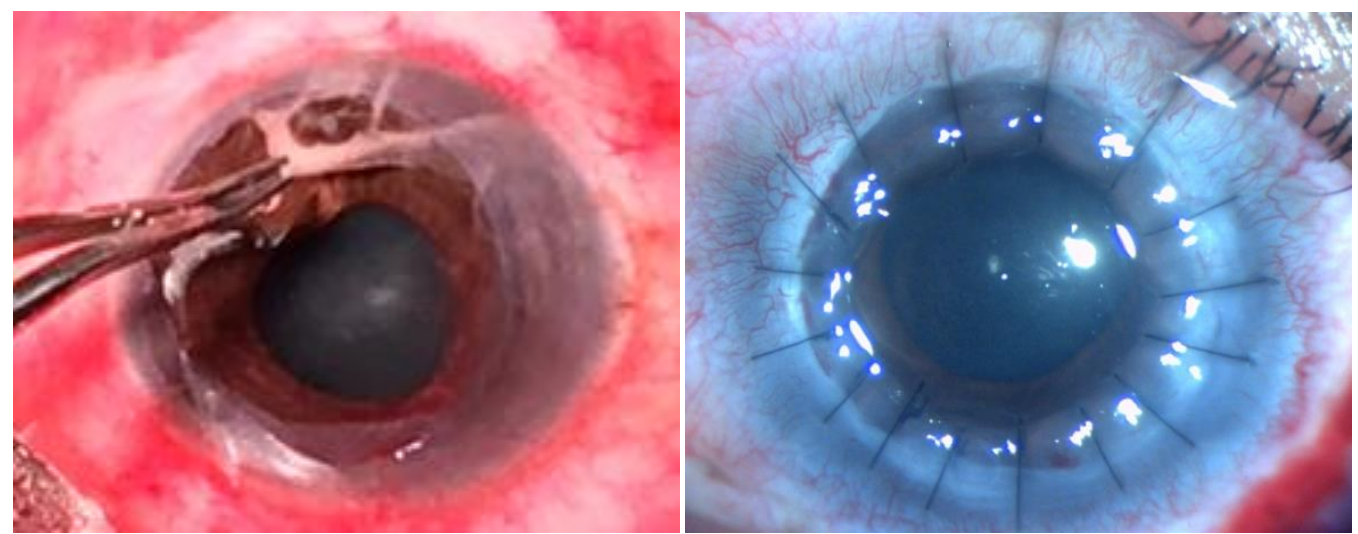

Fig. 1: (a): Removal of exudates from AC (b): Long and tight sutures

\section{Diagnosis}

Post-operative IOP assessment, disc and visual fields examination become difficult affair after PK due to changes in corneal thickness and post-operative astigmatism. Gonioscopy can be done to find out the extent of the PAS. Detailed stereoscopic optic disc evaluation is required. Due to irregular corneal surface in early post-operative period, IOP can be measured independent of the corneal thickness with the Mackay-Marg electronic applanation tonometer, the pneumatic applanation tonometer, tono-pen, or recent dynamic contour tonometer (DCT). ${ }^{10}$ Goldmann applanation tonometre (GAT) can be used only if graft surface epithelium is intact. Prism rotation is done so that the red mark on the prism holder is set at the flatter meridian (negative axis) of the cornea to obtain accurate reading. Average of two readings taken 90 degrees apart can be taken. Corneal edema and corneal opacity reduces the accuracy of GAT. ${ }^{11}$ False low readings will be observed in corneal edema and false high in corneal scarring. Increase in the corneal thickness leads to overestimation of IOP. Proview phosphene tonometer is also available to measure IOP through the lid. Ultrasound biomicroscope (UBM) is useful in opaque corneas to assess the angle and anteriorsegment anatomy where the details are not clearly visible. UBM helps to diagnose relative pupillary block, PAS and plateau iris configuration. It provides an accurate objective quantification of angles, anterior-chamber depth (ACD) assessment, location and extent of iridocorneal adhesions phakic/aphakic status, IOL position, and corneal graft thickness. Quadrant of placement of the tube of the glaucoma drainage device (GDD) in the anterior chamber (AC) can be planned with UBM if details are not visible. In opaque grafts PAS leads to PPKG frequently. ${ }^{12}$ ASOCT is non-contact, non-invasive, high-resolution, real-time modality for detailed visualization of anterior segment with opaque corneal grafts. The longer wavelength light scatters less in opaque tissues, allows deeper penetration and permits imaging through the limbus to visualise angle structures such as the scleral spur and angle recess. The commonly used quantitative parameters are as angle opening distance, angle recess, trabecular iris space area, iris thickness, AC width. ${ }^{13}$ Site of trabeculectomy can be decided preoperatively with ASOCT. In post-operative follow up rapid and high-resolution images of the AC angle, PAS, posterior synechiae (with IOL) and obstruction of an AC drainage tube can be observed. ${ }^{14}$ Complete angle evaluation using ASOCT is recommended in all eyes with PPKG before planning any surgical intervention. Post-operative evaluation of bleb can also be done with AS-OCT. (Fig. $2 a, b, c)$

\section{Management}

PPKG can damage the graft as it leads to enhanced endothelial cells attrition and damage. Therefore, it should be prevented and treated aggressively.

\section{Prophylaxis}

Graft failure is commonly seen in pre-existing glaucoma in aphakic and pseudophakic eyes and is difficult to treat. Reinhard et al reported the 3-year graft survival rate in $71 \%$ patients with a pre-existing glaucoma in contrast to $89 \%$ without such history. ${ }^{15}$ Oversizing of donor graft by 0.5 $1 \mathrm{~mm}$ provides good AC depth and lower incidence of PAS which decreases chances of postoperative glaucoma. ${ }^{16}$ Removal of viscoelastic from AC at the end of the surgery and careful wound closure with deep suturing are the factors which prevent PPKG. Steroids and cycloplegics in postoperative period can decrease inflammation, prevents PAS formation and pupillary block glaucoma.

\section{Medical Treatment}

Beta-adrenergic blocking agents (timolol, betaxolol) reduces the aqueous production. But these drugs can damage the ocular surface by impairing the quantity and quality of the mucus layer of tear film and subsequently leads to dry eye. Corneal anesthesia and dry eye can damage epithelium and eventually it affects the graft clarity. Brimonidine tartrate $0.2 \%$ a selective alpha-2 adrenergic agonist is useful in controlling IOP and is better tolerated but known for its allergic reactions. Miotics are not safe because it can induce uveitis which may initiate graft rejection and increase the risk of a retinal detachment hence not recommended for aphakic eyes. Topical carbonic anhydrase inhibitors like dorzolamide and brinzolamide suppress the carbonic anhydrase enzyme in corneal endothelium and lead to irreversible corneal decompensation. These drugs should not 


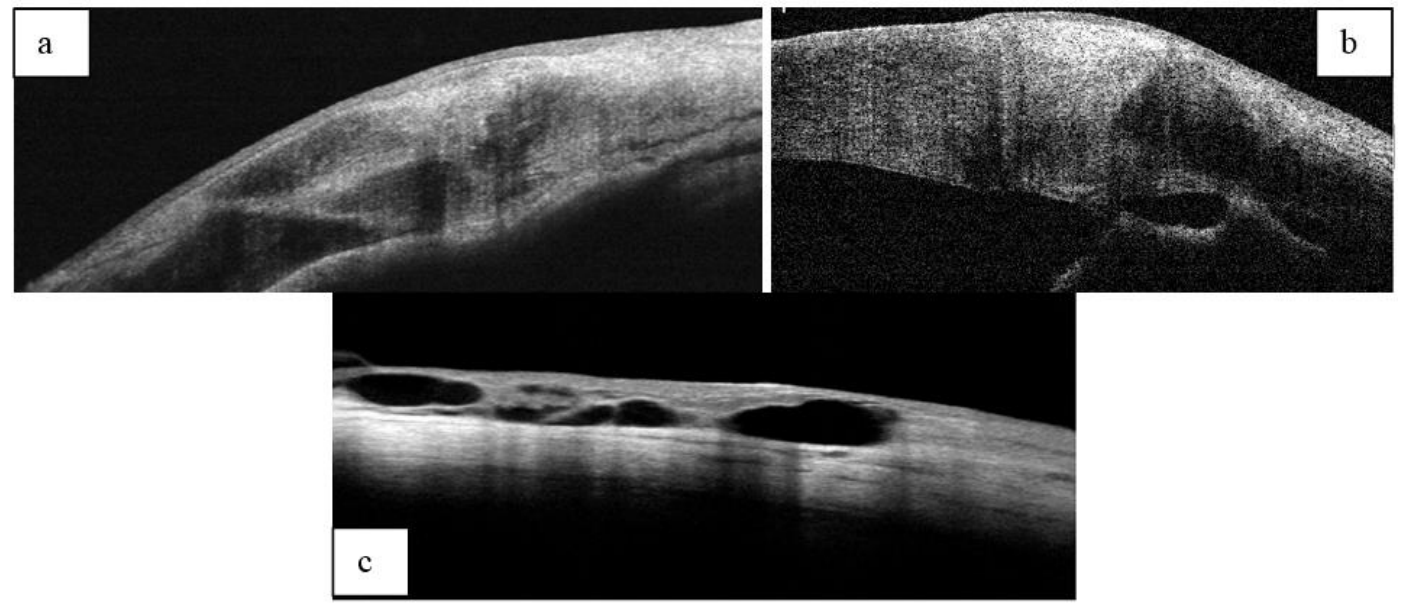

Fig. 2 (a,b): Peripheral anterior synechiae at graft host junction; (c): Demonstrating various aqueous pockets of bleb

be used in patients with a past history of graft rejection or with limited endothelial cell counts. ${ }^{17}$ Systemic carbonic anhydrase inhibitors are useful in the treatment of pressure spikes in the immediate postoperative period. These drugs should be used cautiously in elderly patients because of their side effects such as paresthesias, tinnitus, gastrointestinal disturbances, fatigue, depression, anorexia, and weight loss. ${ }^{18}$

Prostaglandin analogs increase the uveoscleral outflow and decrease IOP. It should not be used in patients with history of herpes simplex keratitis because it can lead to its recurrence. It can induce uveitis and cystoid macular edema in aphakia and pseudophakia. Benzalkonium chloride (BAC $0.01 \%$ concentration) is the preservative used in the majority of these topical medications which can damage corneal epithelium by damaging cell wall and microvilli. Preservative free drugs should be used after PK to protect the corneal epithelium. Post operative topical steroids should be tapered or replaced by least potent steroid to reduce the chances of raised IOP. Topical cyclosporin A $0.5 \%$ may also help to control the pressure, reduces chances of dry eye also. Perry et al reported that after replacement of topical corticosteroids by topical cyclosporin A $0.5 \%$ in 21 $(84 \%)$ of their 25 patients a mean reduction of IOP by 8.7 $\mathrm{mm} \mathrm{Hg}$ was found. Graft clarity was maintained in all patients except one with allograft rejection. ${ }^{19}$

\section{Surgical Treatment}

Laser trabeculoplasty is indicated in patients whose IOP is not controlled with topical medications. Argon laser trabeculoplasty (ALT) is helpful in patients with open angle glaucoma, clear grafts with IOP in range of $20-25 \mathrm{mmHg}$. Van meter reported its success in PPKG in aphakic and pseudophakic eyes. ${ }^{20}$ It is not without limitations. In cases of PAS and poor visibility of the trabecular meshwork it cannot be done. Trabeculectomy is the surgical procedure but it is usually not effective in PPKG because of dense scarring at perilimbal areas, extensive PAS and shallow AC. In aphakic eyes chances of the failure of surgery is further increased due to blockage of the trabeculectomy ostium by the vitreous. The success of surgery can be enhanced by the use of anti-metabolites (5-fluorouracil [5FU] and mitomycin-C[MMC]) by their antifibroblastic activities. Subconjunctival injection of $5 \mathrm{FU}$ with dose of $5 \mathrm{mg}$ in 0.1 $\mathrm{cc}$ is given daily for 7-10 days in the immediate postoperative period for 7-10 days. ${ }^{21}$ Mitomycin-C $0.04 \%$ can be applied for 2-4 min subconjunctivally or sub-sclerally which increases the success rate of filtering surgery. Misaki et al observed $73.0 \%$ success after trabeculectomy with mitomycin $\mathrm{C}$ without any anti-glaucoma medications. Trabeculectomy without mitomycin $\mathrm{C}$ failed within 6 months in most of the cases. Persistent epithelial defect, cystoid macular oedema, choroidal detachment, leaking bleb and graft rejection had been reported..$^{22}$ Ayyala reported $77 \%$ success rate in IOP control with use of mitomycin-C in trabeculectomy patients of PKG. ${ }^{23}$

Raj et al reported successful IOP control post trabeculectomy with releasable suture in PPKG patients till three months as compared to trabeculectomy augmented by MMC. At six months of follow up results in both groups were comparable. ${ }^{24}$ GDD creates a collateral pathway and pushes aqueous from the $\mathrm{AC}$ to an equatorial plate via a tube which leads to bleb formation. The use of GDD in PKG was first to reported by Kirkness in 1987. The risk of graft rejection was high by retrograde passage of the inflammatory cells into the $\mathrm{AC}$ by these devices despite good IOP control. ${ }^{25}$ Post-surgical uveitis, extensive PAS and multiple prior surgeries can lead to graft compromise. ${ }^{26}$ Sherwood reported good IOP control with GDD after two years of follow up i.e upto $96 \% .{ }^{27}$ The location for the tube of device remains controversial. Arroyave found no difference in IOP control between the AC and pars plana tube placement, but $83 \%$ of grafts remained clear with tubes in pars plana as compared to $48 \%$ into AC. Tube into the vitreous cavity significantly reduces its interaction with graft which leads to improvement in graft survival. ${ }^{28}$ Sidoti studied pars plana tube insertion after pars plana vitrectomy and found IOP control in $62 \%$ and clear grafts in $41 \%$ of the eyes after 2 years but was associated with higher rate of posterior segment complications. ${ }^{29}$ Ritterband studied 83 eyes in which the drainage tube was either placed in the pars plana primarily or moved from the $\mathrm{AC}$ to the pars plana at 
the time of PK and reported IOP control in $83 \%$ and graft clarity in $59 \%$ of patients after 2 years. ${ }^{30}$ Shunt tubes can be occluded in into the vitreous cavity so complete vitrectomy with removal of the vitreous skirt is mandatory to prevent its occlusion. Well-placed tube in the AC away from the cornea allows its observation at the slit lamp and easy access for laser and needling to remove any tissues if the tube tip is blocked Cyclodestructive procedures can be employed to lower IOP by decreasing aqueous humour production by ablating the portion of ciliary body. Destruction of the ciliary body can be achieved through transscleral application of cryotherapy or transscleral or endoscopic delivery of diode, krypton or Nd:YAG laser.. Recent studies showed success rate of $72 \%$ after an year of single treatment of diode laser in terms of improvement in visual acuity, no graft rejection and minimal anti-glaucoma medications. No complications were noticed in form of phthisis bulbi or graft failure. $^{31}$

\section{Conclusions}

Graft survival and permanent irreversible visual loss due to glaucoma are two important factors to be managed in PPKG. Knowledge of the various risk factors such as preexisting glaucoma, pseudophakia, aphakia and repeat PK may increase the chances of success of PK. Avoidance of predisposing factors, timely diagnosis of PPKG along with aggressive and timely management remains the cornerstone for preserving optimal graft clarity and visual function following PK.

\section{Acknowledgements}

Mr. Surendra Singh Bhandari, Office Assistant for his help in documentation.

\section{Financial Support: Nil}

\section{Conflict of Interest: None}

\section{References}

1. Ayyala RS. Penetrating keratoplasty and glaucoma. Surv Ophthalmol 2000;45:91-105.

2. Foulks GN. Glaucoma associated with penetrating keratoplasty. Ophthalmol 1987;94,871-4.

3. Hemanth Raj MN, Bhanushree G, Hlinaykor RM, Vijayanath V. Preoperative risk factors and incidence of glaucoma after penetrating keratoplasty. Int J Clin Trials 2014;1:55-61.

4. Franea ET, Araeri ES, Roche FJ. A study of glaucoma after penetrating keratoplasty. Cornea 2002;1:284-8.

5. Raj A, Dhasmana R, Bahadur H, "Incidence and risk factors for post keratoplasty glaucoma in tertiary care centre, India. Oman J Ophthalmol 2018;11:220-6.

6. Karadag O, Kugu S, Erdogan G, Kandemir B, Ozdil S E, Dogan O K. Incidence of and Risk Factors for Increased Intraocular Pressure After Penetrating Keratoplasty. Cornea 2010;29,278-82.

7. Olson RJ, Kaufman HE. A mathematical description of causative factors and prevention of elevated intraocular pressure after keratoplasty. Invest Ophthalmol Vis Sci 1977;16:1085-92.
8. Zimmerman TJ, Krupin T, Grodzki W, Waltman SR. The effect of suture depth on outflow facility in penetrating keratoplasty. Arch Ophthalmol 1978;96:505-6.

9. Zimmerman T, Olson R, Waltman S, Kaufman H. Transplant size and elevated intraocular pressure. Postkeratoplasty. Arch Ophthalmol 1978;96:2231-3.

10. McMillan F, Forster RK. Comparison of MacKay-Marg, Goldmann, and Perkins tonometers in abnormal corneas. Arch Ophthalmol 1975;93:420-4.

11. Doughty MJ, Zaman ML. Human corneal thickness and its impact on intraocular pressure measures: a review and metaanalysis approach. Surv Ophthalmol 2000;44:367-408.

12. Dada T, Aggarwal A, Vanathi M, Gadia R, Panda A, Gupta V. Ultrasound biomicroscopy in opaque grafts with postpenetrating keratoplasty glaucoma. Cornea 2008;27:402-5.

13. M.Farnaz, L Yan, F A Brian, S E Ronald, G Julie. Optical coherence tomography of anterior segment in secondary glaucoma with corneal opacity after penetrating keratoplasty. Br J Ophthalmol 2007;91:189-92.

14. Dada T, Shah B M, Bali S J, Bansal N, Panda A and Vanathi M. Anterior segment OCT imaging in opaque graft with secondary glaucoma following tectonic penetrating keratoplasty for perforated corneal ulcer. Eye 2011;25:1522-4.

15. Reinhard T, Kallmann C, Cepin A. The influence of glaucoma history on graft survival after penetrating keratoplasty. Graefes Arch Clin Exp Ophthalmol 1997;235:553-7.

16. Vajpaye R B, Dada T, Ray M, Tandon R, Sethi A, Turaka. Oversized corneal grafts for corneal opacities with iridocorneal adhesions. Ophthalmol 2011;108:2026-8.

17. Konowal A, Morrison JC, Brown SV. Irreversible corneal decompensation in patients treated with topical dorzolamide. Am J Ophthalmol 1999;127:403-6.

18. Epstein DL, Bramsen T, Sperling S. Carbonic anhydrase inhibitor side effects. Serum chemical analysis. Arch Ophthalmol 1977;95:1378-82.

19. Perry HD, Donnenfeld ED, Kanellopoulos AJ. Topical cyclosporin A in the management of postkeratoplasty glaucoma. Cornea 1997;16:284-8.

20. Van Meter WS, Allen RC, Waring CO. Laser trabeculoplasty for glaucoma in aphakic and pseudophakic eyes after penetrating keratoplasty. Arch Ophthalmol 1988;106:185-8.

21. Skuta GL, Beeson CC, Higginbotham EJ. Intraoperative mitomycin versus postoperative 5-fluorouracil in high risk glaucoma filtering surger. Ophthalmol 1992;99:438-44.

22. Ishiokaa M, Shimazakia J, Fujishimaa H, Shimmuraa S, Tsubotaa K. Trabeculectomy with mitomycin C for postkeratoplasty glaucoma. Br J Ophthalmol 2000;84:714-7.

23. Ayyala RS, Pieroth L, Vinals AF, "Comparison of mitomycin $\mathrm{C}$ trabeculectomy, glaucoma drainage device implantation, and laser neodymium: YAG cyclophotocoagulation in the management of intractable glaucoma after penetrating keratoplasty. Ophthalmol 1998;105:1550-6.

24. Raj A, Dhasmana R, Bahadur H. Comparative evaluation of trabeculectomy with releasable suture versus subconjunctival Mitomycin C in post keratoplasty glaucoma". Sudanese J Ophthalmol 2017;9:16-21.

25. Kirkness CM, "Penetrating keratoplasty, glaucoma and silicone drainage tubing. Dev Ophthalmol 1987;14:161-5.

26. Kirkness CM, Ling Y, Rice NS, The use of silicone drainage tubing to control post-keratoplasty glaucoma. Eye 1988;2:58390.

27. Sherwood MB, Smith MF, Driebe Jr WT, Stern GA, Beneke JA, Zam ZS. Drainage tube implants in the treatment of glaucoma following penetrating keratoplasty. Ophthalmic Surg 1993;24:185-9.

28. Arroyave CP, Scott IU, Fantes FE, Feuer WJ, Murray TG. Corneal graft survival and intraocular pressure control after 
penetrating keratoplasty and glaucoma drainage device implantation. Ophthalmol 2001;108:1978-85.

29. Sidoti PA, Mosny AY, Ritterband DC, Seedor JA. Pars plana tube insertion of glaucoma drainage implants and penetrating keratoplasty in patients with coexisting glaucoma and corneal disease. Ophthalmol 2001;108:1050-8.

30. Ritterband DC, Shapiro D, Trubnik V, Marmor M, Meskin S, Seedor J. Cornea Glaucoma Implant Study Group (COGIS). Penetrating keratoplasty with pars plana glaucoma drainage device. Cornea 2007;26:1060-6.

31. Ocakoglu O, Arslan OS, Kayiran A. Diode laser transscleral cyclophotocoagulation for the treatment of refractory glaucoma after penetrating keratoplasty. Curr Eye Res 2005;30:569-54.

How to cite this article: Raj A, Bahadur H. Glaucoma after penetrating keratoplasty: Risk factors and its management. Int $J$ Ocul Oncol Oculoplasty $2019 ; 5(2): 44-8$ 